\title{
Seven Dimensional New Hyperchatic Systems: Dynamics and Synchronization by a High Gain Observer Design
}

\author{
S.N. Lagmiri", M. Amghar and N. Sbiti \\ Electrical Engineering Department \\ Mohammadia School Engineering \\ University Mohammed V-Rabat - MOROCCO \\ najoua.lagmiri@gmail.com ${ }^{*}$,amghar@emi.ac.ma, sbiti@emi.ac.ma
}

\begin{abstract}
The aim of this paper is to synchronize the two new hyperchaotic systems that we have developped. These new systems are the 7-dimensional fourth-order and six-order continuous real hyperchaotic systems. After studying and verifying the hyperchaotic bihaviour of these systems, a class of high gain observer is used to synchronize and to stabilize the synchronization error dynamic. The stability of the systems is proven using Lyapunov stability theory. Simulations results confirm the high performance of the designed observer for these high dimensional systems.
\end{abstract}

Keyword: Synchronization, 7D fourth-order hyperchaotic system, 7D six-order hyperchaotic system, Equilibrium point, Lyapunov exponent, High gain observer.

\section{Introduction}

In recent years, the chaotic system has been used more and more widely in secure communication with the further research on the chaos theory. Compared to other methods, the encryption method has such advantages as good security, strong dynamic storage capability and low power, which significantly improves the safety and reliability of information transmission. When the common chaotic system signal is used as the encrypted signal, the message can be deciphered and attacked easily. However, the characteristics of the hyperchaotic system are more complex. Thus, to use the hyperchaotic system signal as the encrypted signal has more extensive application prospect [1-4]. In [5-7], the four-dimensional system, the six-dimensional system and their realizing circuits are given, which lays a foundation of the construction of the higher dimensional hyperchaotic system.

When we take an ordinary chaotic systems signal as an encrypted signal, the information can easily be deciphered and attacked, and because the chaotic characteristic of hyperchaotic system is more complex, so hyperchaotic system as information encrypted signal has a broader application prospect.

The goal of this paper is to construct two new 7D hyperchaotic systems and to investigate the dynamics and synchronization of these new systems using the theory of observers. In this approach, once the drive system is given, the response system could be chosen in the observer form, and the drive signal should be chosen accordingly so that the drive system satisfies certain conditions.

The outline of this paper is as follows. In Section 2, we set the problem statement and the adopted methodology. In Section 3, we expose the high gain observer that we used to synchronize our hyperchaotic systems. In section 4, we define our 7D fourth-order and six-order hyperchaotic systems. In Section 5, the synchronization problem is formulated.

${ }^{*}$ Corresponding Author 
And in section 6, we derive results for the drive-response synchronization of the two 7D hyperchaotic systems. Conclusion is presented in the last section.

\section{Research Method}

Consider the hyperchaotic system described by the dynamic:

$$
\dot{x}=A x+f(x)
$$

Where $x \in R^{n}$ is the state of the system, $A$ is the $n \times n$ matrix of the system parameters and $f: R^{n} \rightarrow R^{n}$ is the nonlinear part of the system.

We consider the system (1) as the drive system.

As the response system, we consider the following hyperchaotic system described by the dynamic:

$$
\dot{y}=B y+g(y)+K(\theta)
$$

Where $y \in R^{n}$ the state of the system, B is the $n \times n$ matrix of the system parameters and $g: R^{n} \rightarrow R^{n}$ is the nonlinear part of the system and $\theta$ is the gain of the response system.

- If $A=B$ and $f=g$, then $x$ and $y$ are the states of two identical hyperchaotic systems.

- If $A \neq B$ or $f \neq g$, then $x$ and $y$ are the states of two different hyperchaotic systems.

In the nonlinear feedback control approach, we design an observer, which synchronizes the states of the drive system (1) and the response system (2) for all initial conditions $x(0), y(0) \in R^{n}$.

If we define the synchronization error as:

$$
e=y-x
$$

Thus, the synchronization problem is essentially to find a controller $\theta$ so as to stabilize the error $e$ for all initial conditions $e(0) \in R^{n}$.

In our case $\theta$ is a high gain observer.

\section{High Gain Observer Design}

The problem of observer design naturally arises in a system approach, as soon as one needs unmeasured internal information from external measurements. In general, it is clear that one cannot use as many sensors as signals of interest characterizing the system behavior for technological constraints, cost reasons, and so on, especially since such signals can come in a quite large number, and they can be of various types: they typically include parameters, time-varying signals characterizing the system (state variables), and unmeasured external disturbances $[9,3,20]$. As we know, it is almost impossible to measure all the elements of the state vector in practice (e.g., the unknown state variables, fault signals, etc).

State observers are able to provide a continuous estimation of some signals which are not measured by hardware sensors. They need a mathematical model of the process and hardware measurements of some other signals. An observer is a dynamic system whose input includes the control $u$ and the output $y$ and whose output is an estimate of the state vector $\hat{x}$ as it's shown in Figure1. 


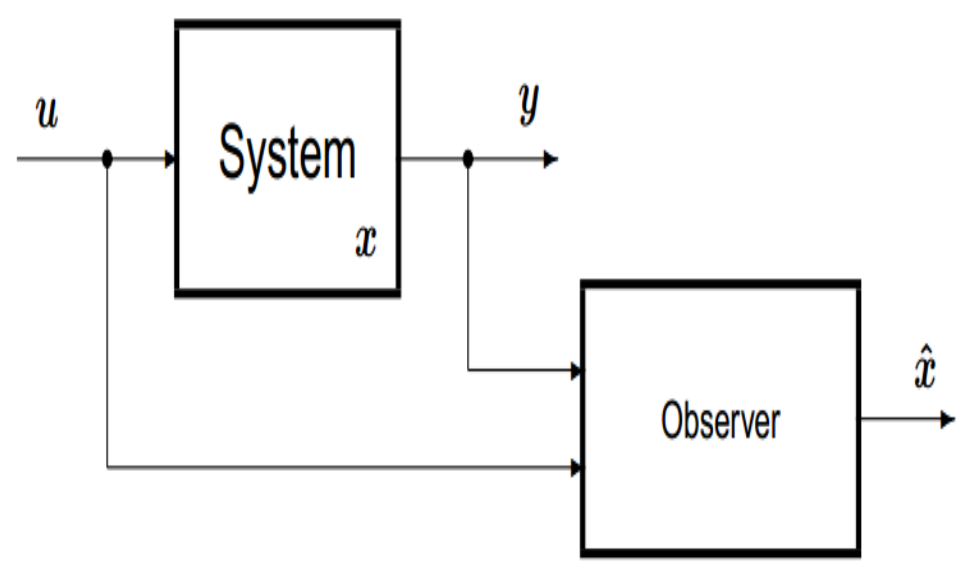

Figure 1. Principle of the Observer

We consider a general representation of the hyperchaotic system as follow:

$\left\{\begin{array}{c}\dot{x}=f(x) \\ y=C x\end{array}\right.$

Where:

- $\quad C=\left(\begin{array}{llll}1 & 0 & \ldots & 0\end{array}\right)$ and $x \in R^{n}$ is the vector of states, $x=\left(x_{1}, x_{2}, \ldots, x_{n}\right)^{T}$.

- $\quad f: R^{n} \rightarrow R^{n}$ is the nonlinear part of the system.

- $\quad y \in R^{m}$ is the system measured output with $m<n$, and

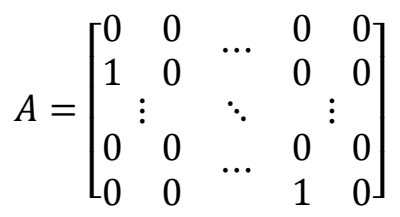

It is necessary the design of an auxiliary system so called observer system to reconstruct then known states or unmeasurables. Firstly, we give necessary and sufficient conditions to establish whether the system (4) is observable.

Now, consider the following assumptions [5]:

\section{A. Assumptions}

A1. The system given in Eqs (4) is locally uniformly observable (Gauthier et al., 1992), hence for all $x \in R^{n}$, satisfies the observability rank condition:

$$
\operatorname{rang}\left\{\frac{\partial}{\partial x} \vartheta\right\}=n
$$

Here $\vartheta$ is the observability vector function define $\mathrm{d} \vartheta=\left(d L_{f}^{0} h, d L_{f}^{1} h, \ldots, d L_{f}^{n-1} h\right)^{T} L_{f}^{r}$ the r-order Lie derivatives, which are the directional derivatives of the corresponding state variables along the measured output trajectory. And $d L_{f}^{r} h$ are the differentials of the rthorder Lie derivatives defined recursively as follows:

$$
\begin{aligned}
& L_{f}^{0} h:=h, d L_{f}^{0} h:=d h=\left(\frac{\partial h}{\partial x_{1}}, \ldots, \frac{\partial h}{\partial x_{n}}\right) \\
& L_{f}^{1} h:=<d h, f>=\sum_{i=1}^{n} \frac{\partial h}{\partial x_{i}} f_{i} \\
& d L_{f}^{1} h:=\left(\frac{\partial}{\partial x_{1}}\left(\sum_{i=1}^{n} \frac{\partial h}{\partial x_{i}} f_{i}\right), \ldots, \frac{\partial}{\partial x_{n}}\left(\sum_{i=1}^{n} \frac{\partial h}{\partial x_{i}} f_{i}\right)\right)
\end{aligned}
$$


$L_{f}^{r} h:=<d L_{f}^{r-1} h, f>=L_{f}\left(L_{f}^{r-1} h\right), \quad r \geq 2$

A2. All the trajectories $x\left(t, x_{0}\right), x_{0} \in R^{n}$ of the system (4) are bounded.

Considering the set $\Omega \subset R^{n}$ as the corresponding physically realizable domain, such that:

$\Omega=\left\{\left(x_{i}\right)_{i=1}^{n} \in R_{+}^{n} / 0 \leq x_{i} \leq x_{\max }\right\}$

In most practical cases, $\Omega$ will be an open connected relatively compact subset of $R^{n}$, and in the ideal cases, $\Omega$ will be positively invariant under the dynamic (4).

In order to analyze the estimation error $\varepsilon=y-x$ we consider the next assumption.

A3. The nonlinear difference vector function $\Delta \emptyset=f(x)-f(\hat{x})$ is Lipschitz bounded i.e. $\Delta \emptyset<\Lambda|\varepsilon|$.

Condition A3 can be fulfilled satisfied if the following supremumis finite: $\Lambda:=\sup _{x \in \Omega}\left\|f^{\prime}(x)\right\|$

Where $f^{\prime}(x)$ is the Jacobian and $\|$.$\| is the matrix norm associated with the Euclidian$ vector norm.

\section{B. Propositions}

For any initial condition $x(0) \in D$ and any $\hat{x}(0) \in D$ and for $\theta$ large enough the system (1) satisfying previous assumptions [10] [11], can be estimated by the following dynamical system:

$$
\dot{\hat{x}}=f(\hat{x})-\theta * d_{\theta}^{-1} * S_{1}^{-1} * C^{\prime}(C \hat{x}-y)
$$

Where $S_{1}$ is the symetric positive definite solution of the algebraic equation:

$\theta S_{\theta}+A^{\prime} S_{\theta}+S_{\theta} A-C^{\prime} C=0$

For $\theta=1$ and it can be expressed as

$S_{1}(i, j)=(-1)^{i+j} C_{i+j-2}^{j-1}$, for $1 \leq i, j \leq n$

where $C_{j}^{i}=\frac{j !}{i !(j-i) !}$

$d_{\theta}$ is a diagonal matrix defined by :

$d_{\theta}=\operatorname{diag}\left(1, \frac{1}{\theta}, \ldots, \frac{1}{\theta^{n}}\right)$

\section{Lemma}

For $\theta$ large enough the slave system below is an observer for the drive system [15]:

$$
\dot{\hat{x}}=f(\hat{x})-\theta * d_{\theta}^{-1} * S_{1}^{-1} * C^{\prime}(C \hat{x}-z)
$$

\section{Proof [10]}

Let $e=\hat{x}-x$

Then on can check that:

$\dot{e}=\left(A-\theta d_{\theta}^{-1} S_{1}^{-1} C^{T} C\right) e+\Delta F$

Where:

$\Delta F=F(\hat{x})-F(x)$

$\mathrm{F}$ is lipschitz with the constant $\mathrm{L}$ so:

$\|\Delta F\| \leq L\|e\|$

Let $V_{\theta}$ a candidate lyapunov equation for the system (1):

$V_{\theta}=\frac{1}{\theta} e^{\prime} d_{\theta} S_{1} d_{\theta} e$ 
The time derivative of $V_{\theta}$ computed along solution of the differential equations (1) is given by:

$\dot{V}_{\theta}=\frac{1}{\theta} e^{\prime} d_{\theta}\left(d_{\theta}^{-1} A^{\prime} d_{\theta} S_{1}+S_{1} d_{\theta} A d_{\theta}^{-1}-\theta d_{\theta}^{-1} C^{\prime} C-\theta C^{\prime} C d_{\theta}^{-1}\right) d_{\theta} e+2 \frac{1}{\theta} \Delta(F) d_{\theta} S_{1} d_{\theta} e$

Taking into account the algebraic equation:

$\theta S_{\theta}+A^{\prime} S_{\theta}+S_{\theta} A-C^{T} C=0$

And

$d_{\theta} A d_{\theta}^{-1}=\theta A, C^{T} C d_{\theta}^{-1}=C^{T} C$

It follows

$\dot{V}_{\theta}=\frac{1}{\theta} e^{\prime} d_{\theta}\left(-\theta S_{1}-\theta C^{\prime} C\right) d_{\theta} e+2 \frac{1}{\theta} \Delta(F) d_{\theta} S_{1} d_{\theta} e$

Using the above inequality:

$\lambda_{\min }\left(S_{1}\right)\left\|d_{\theta} e\right\|^{2} \leq e^{\prime} d_{\theta} S_{1} d_{\theta} e \leq \lambda_{\max }\left(S_{1}\right)\left\|d_{\theta} e\right\|^{2}$

Where $\lambda_{\min }\left(S_{1}\right)$ and $\lambda_{\max }\left(S_{1}\right)$ are respectively the minimum and the maximum eigenvalues of $S_{1}$.

\section{Systems Description}

\subsection{Seven-Dimensional Fourth-Order Hyperchaotic System}

In this section, we design the 7D fouth-order hyperchaotic system that is token as the drive system, whose dynamics is given by:

$$
\begin{aligned}
& \dot{\mathrm{x}}_{1}=-\mathrm{ax}_{1}-20 \mathrm{x}_{5}-\mathrm{x}_{3} \mathrm{x}_{5} \mathrm{x}_{6} \mathrm{x}_{7} \\
& \dot{\mathrm{x}}_{2}=\mathrm{cx}_{2}-\mathrm{dx}_{6}+\mathrm{x}_{1} \mathrm{x}_{4} \mathrm{x}_{5} \mathrm{x}_{7} \\
& \dot{\mathrm{x}}_{3}=-\mathrm{ax}_{3}+\mathrm{ax}_{5}-\mathrm{g} \mathrm{x}_{1} \mathrm{x}_{2} \mathrm{x}_{4} \mathrm{x}_{6} \\
& \dot{\mathrm{x}}_{4}=-\mathrm{ax}_{4}+\mathrm{ex}_{1}+\mathrm{x}_{1} \mathrm{x}_{2} \mathrm{x}_{3} \mathrm{x}_{5} \\
& \dot{\mathrm{x}}_{5}=-\mathrm{ax}_{5}+\mathrm{ex}_{7}-\mathrm{x}_{1} \mathrm{x}_{2} \mathrm{x}_{3} \mathrm{x}_{4} \\
& \dot{\mathrm{x}}_{6}=-\mathrm{ex}_{6}+\mathrm{ex}_{5}+\mathrm{x}_{2} \mathrm{x}_{3} \mathrm{x}_{4} \mathrm{x}_{5} \\
& \dot{\mathrm{x}}_{7}=-\mathrm{bx}_{7}+\mathrm{fx}_{2}-\mathrm{hx}_{1} \mathrm{x}_{4} \mathrm{x}_{5} \mathrm{x}_{6}
\end{aligned}
$$

Where $a, b, c, d, e, f, g$ and $h$ are the parameters of the system and $x \in R^{n}$ is the state of the system.

$$
a=15, b=5, c=0.5, d=25, e=10, f=4, g=0.1 \text { and } h=1.5
$$

\subsubsection{Dissipation}

The divergence of the system (8) is:

$$
\begin{aligned}
\nabla V= & \frac{\partial \dot{x}_{1}}{\partial x_{1}}+\frac{\partial \dot{x}_{2}}{\partial x_{2}}+\frac{\partial \dot{x}_{3}}{\partial x_{3}}+\frac{\partial \dot{x}_{4}}{\partial x_{4}}+\frac{\partial \dot{x}_{5}}{\partial x_{5}}+\frac{\partial \dot{x}_{6}}{\partial x_{6}}+\frac{\partial \dot{x}_{7}}{\partial x_{7}} \\
& =-a+c-a-a-a-e-b
\end{aligned}
$$

According to the ranges of the system parameters, we obtain that

$-a+c-a-a-a-e-b<0$, and thus the system is a dissipative system.

\subsubsection{Equilibrium and Lyapunov Exponents}

The following equations are the equilibrium of the system satisfied: 


$$
\begin{aligned}
& -\mathrm{ax}_{1}-20 \mathrm{x}_{5}-\mathrm{x}_{3} \mathrm{x}_{5} \mathrm{x}_{6} \mathrm{x}_{7}=0 \\
& \mathrm{cx}_{2}-\mathrm{dx_{6 }}+\mathrm{x}_{1} \mathrm{x}_{4} \mathrm{x}_{5} \mathrm{x}_{7}=0 \\
& -a x_{3}+a x_{5}-g x_{1} x_{2} x_{4} x_{6}=0 \\
& -\mathrm{ax}_{4}+\mathrm{ex}_{1}+\mathrm{x}_{1} \mathrm{x}_{2} \mathrm{x}_{3} \mathrm{x}_{5}=0 \\
& -\mathrm{ax}_{5}+\mathrm{ex}_{7}-\mathrm{x}_{1} \mathrm{x}_{2} \mathrm{x}_{3} \mathrm{x}_{4}=0 \\
& \text { - e } x_{6}+e x_{5}+x_{2} x_{3} x_{4} x_{5}=0 \\
& \text { - } \mathrm{bx}_{7}+\mathrm{fx}_{2}-\mathrm{hx_{1 }} \mathrm{x}_{4} \mathrm{x}_{5} \mathrm{x}_{6}=0
\end{aligned}
$$

The new hyperchaotic system (8) has unique equilibrium point $\mathrm{E}_{0}(0,0,0,0,0,0,0)$. For this latter, the system (8) is linearized, and the Jacobian matrix is given by :

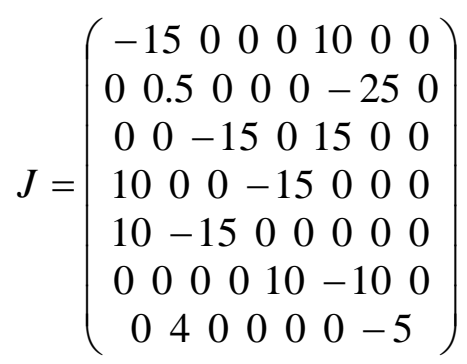

To gain its eigenvalues, let $\left|\lambda_{i}-J_{0}\right|=0$, Then these eigenvalues corresponding to equilibrium $E_{0}(0,0,0,0,0,0,0)$ will be obtained as follows:

$\lambda_{1}=-14.890, \lambda_{2}=0.841, \lambda_{3}=0.489, \lambda_{4}=-14.957, \lambda_{5}=-14.623$, $\lambda_{6}=-15.713, \lambda_{7}=-15.595$

Where $\lambda_{1}, \lambda_{4}, \lambda_{5}, \lambda_{6}, \lambda_{7}$ are negative real roots and $\lambda_{2}, \lambda_{3}$ are positive real roots.

Therefore, equilibrium $\mathrm{E}_{0}$ is unstable.

System (8) has two positive lyapunov exponents, which shows that the system is not only chaotic but also hyperchaotic.

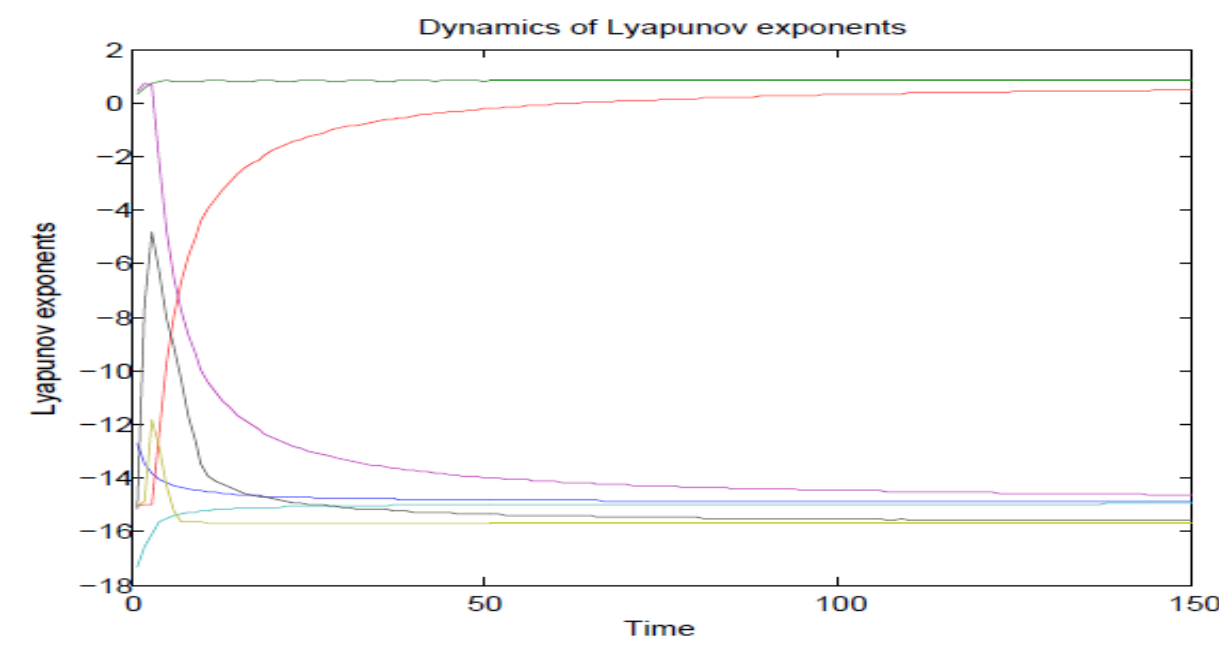

Figure 2. Lyapunov Exponent Seven-Dimensional Fourth-Order Hyperchaotic System

\subsection{Seven-Dimensional Six-Order Hyperchaotic System}

In this section, we design the 7D six-order hyperchaotic system described by the following dynamic: 


$$
\begin{aligned}
& \dot{\mathrm{z}}_{1}=15 \mathrm{z}_{1}+20 \mathrm{z}_{3}+0.785 \mathrm{z}_{2} \mathrm{z}_{3} \mathrm{z}_{4} \mathrm{z}_{5} \mathrm{z}_{6} \mathrm{z}_{7} \\
& \dot{\mathrm{z}}_{2}=-20 \mathrm{z}_{1}-20 \mathrm{z}_{2}-\mathrm{z}_{1} \mathrm{z}_{3} \mathrm{z}_{4} \mathrm{z}_{5} \mathrm{z}_{6} \mathrm{z}_{7} \\
& \dot{\mathrm{z}}_{3}=20 \mathrm{z}_{4}-15 \mathrm{z}_{3}+20 \mathrm{z}_{5}+\mathrm{z}_{1} \mathrm{z}_{2} \mathrm{z}_{4} \mathrm{z}_{5} \mathrm{z}_{6} \mathrm{z}_{7} \\
& \dot{\mathrm{z}}_{4}=5 \mathrm{z}_{4}-\mathrm{z}_{1} \mathrm{z}_{2} \mathrm{z}_{3} \mathrm{z}_{5} \mathrm{z}_{6} \mathrm{z}_{7} \\
& \dot{\mathrm{z}}_{5}=-30 \mathrm{z}_{5}+19 \mathrm{z}_{3}+\mathrm{z}_{1} \mathrm{z}_{2} \mathrm{z}_{3} \mathrm{z}_{4} \mathrm{z}_{6} \mathrm{z}_{7} \\
& \dot{\mathrm{z}}_{6}=10 \mathrm{z}_{2}+3.9 \mathrm{z}_{6}-\mathrm{z}_{1} \mathrm{z}_{2} \mathrm{z}_{3} \mathrm{z}_{4} \mathrm{z}_{5} \mathrm{z}_{7} \\
& \dot{\mathrm{z}}_{7}=20 \mathrm{z}_{3}-15 \mathrm{z}_{7}+1.5 \mathrm{z}_{1} \mathrm{z}_{2} \mathrm{z}_{3} \mathrm{z}_{4} \mathrm{z}_{5} \mathrm{z}_{6}
\end{aligned}
$$

\subsubsection{Dissipation}

The divergence of the system (8) is

$$
\begin{aligned}
\nabla V= & \frac{\partial \dot{z}_{1}}{\partial z_{1}}+\frac{\partial \dot{z}_{2}}{\partial z_{2}}+\frac{\partial \dot{z}_{3}}{\partial z_{3}}+\frac{\partial \dot{z}_{4}}{\partial z_{4}}+\frac{\partial \dot{z}_{5}}{\partial z_{5}}+\frac{\partial \dot{z}_{6}}{\partial z_{6}}+\frac{\partial \dot{z}_{7}}{\partial z_{7}} \\
& =-15-20-15+5-30+3.9-15=-86.1
\end{aligned}
$$

$-86.1<0$, and thus the system is a dissipative system.

\subsubsection{Equilibrium and Lyapunov Exponents}

The following equations are the equilibrium of the system satisfied:

$$
\begin{array}{r}
15 \mathrm{z}_{1}+20 \mathrm{z}_{3}+0.785 \mathrm{z}_{2} \mathrm{z}_{3} \mathrm{z}_{4} \mathrm{z}_{5} \mathrm{z}_{6} \mathrm{z}_{7}=0 \\
-20 \mathrm{z}_{1}-20 \mathrm{z}_{2}-\mathrm{z}_{1} \mathrm{z}_{3} \mathrm{z}_{4} \mathrm{z}_{5} \mathrm{z}_{6} \mathrm{z}_{7}=0 \\
20 \mathrm{z}_{4}-15 \mathrm{z}_{3}+20 \mathrm{z}_{5}+\mathrm{z}_{1} \mathrm{z}_{2} \mathrm{z}_{4} \mathrm{z}_{5} \mathrm{z}_{6} \mathrm{z}_{7}=0 \\
5 \mathrm{z}_{4}-\mathrm{z}_{1} \mathrm{z}_{2} \mathrm{z}_{3} \mathrm{z}_{5} \mathrm{z}_{6} \mathrm{z}_{7}=0 \\
-30 \mathrm{z}_{5}+19 \mathrm{z}_{3}+\mathrm{z}_{1} \mathrm{z}_{2} \mathrm{z}_{3} \mathrm{z}_{4} \mathrm{z}_{6} \mathrm{z}_{7}=0 \\
10 \mathrm{z}_{2}+3.9 \mathrm{z}_{6}-\mathrm{z}_{1} \mathrm{z}_{2} \mathrm{z}_{3} \mathrm{z}_{4} \mathrm{z}_{5} \mathrm{z}_{7}=0 \\
20 \mathrm{z}_{3}-15 \mathrm{z}_{7}+1.5 \mathrm{z}_{1} \mathrm{z}_{2} \mathrm{z}_{3} \mathrm{z}_{4} \mathrm{z}_{5} \mathrm{z}_{6}=0
\end{array}
$$

The new hyperchaotic system (10) has unique equilibrium point $E_{0}(0,0,0,0,0,0,0)$. For this latter, the system (10) is linearized, and the Jacobian matrix is given by :

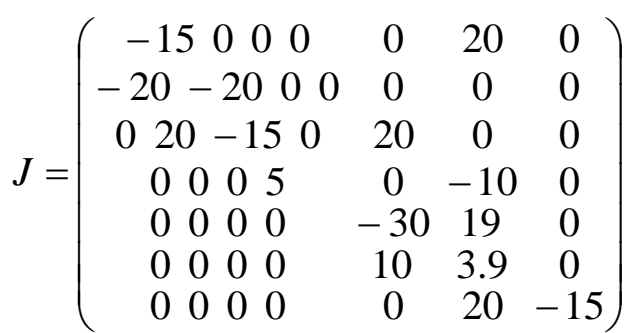

To gain its eigenvalues, let $\left|\lambda_{i}-J_{0}\right|=0$, Then these eigenvalues corresponding to equilibrium $E_{0}(0,0,0,0,0,0,0)$ will be obtained as follows:

$\lambda_{1}=3.894, \quad \lambda_{2}=-14.927, \quad \lambda_{3}=-1.610, \quad \lambda_{4}=5.001, \lambda_{5}=-14.943$, $\lambda_{6}=-10.951, \lambda_{7}=-10.238$

Where $\lambda_{2}, \lambda_{3}, \lambda_{5}, \lambda_{6}, \lambda_{7}$ are negative real roots and $\lambda_{1}, \lambda_{4}$ are positive real roots.

Therefore, equilibrium $\mathrm{E}_{0}$ is unstable.

System (10) has three positive lyapunov exponents, which shows that the system is not only chaotic but also hyperchaotic. 


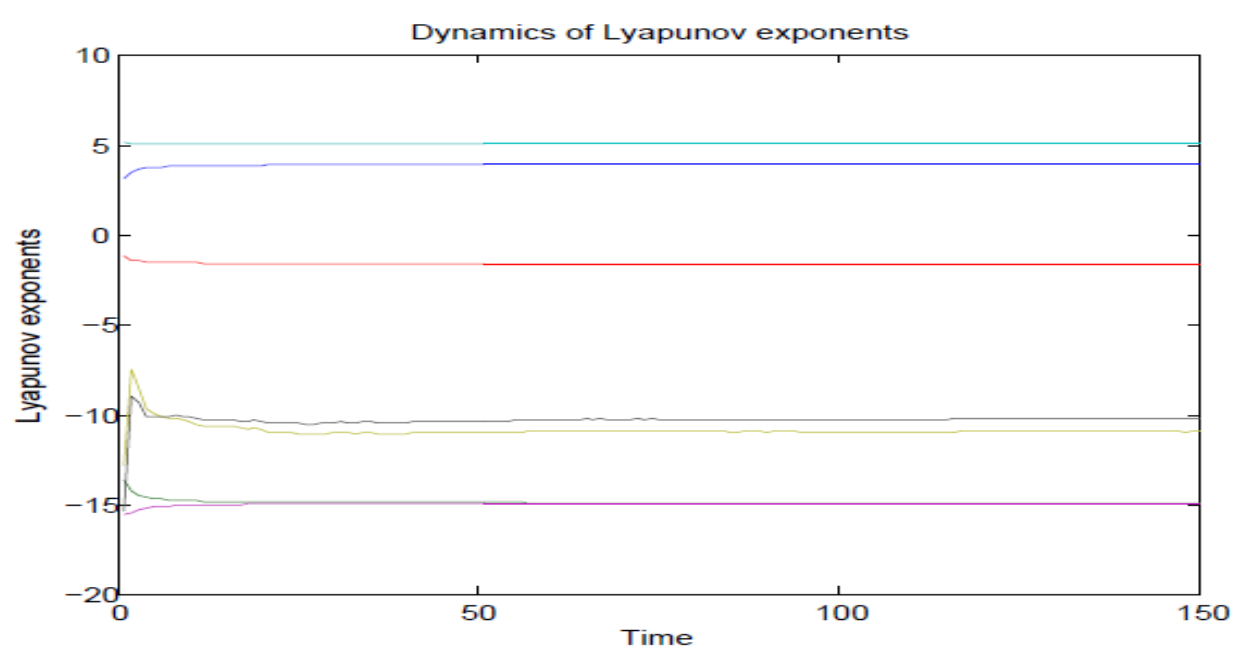

Figure 3. Lyapunov Exponent Seven-Dimensional Six-Order Hyperchaotic System

\section{Synchronization Problem Formulation}

As reported in the literature, synchronization of hyperchaotic systems suggests the possibility for communication using hyperchaotic waveforms as carriers, perhaps with application to secure communication. The obvious approach uses a hyperchaotic system as the transmitter and a synchronous hyperchaotic system for the receiver, and several designs have been suggested that fit within this construct [8]. The variation in these designs lies in the methods for injecting an information signal at the transmitter and recovering it at the receiver.

Definition: Two hyperchaotic systems are completely synchronized if the error [9]: $\|\hat{x}-x\| \rightarrow 0$ as $t \rightarrow \infty$

It means that each state in the slave system is identical or is very close to its corresponding state in the master system along the time.

Chaos synchronization as stabilization consists in finding a control command that leads the trajectories of a dynamical error system to the origin [17].

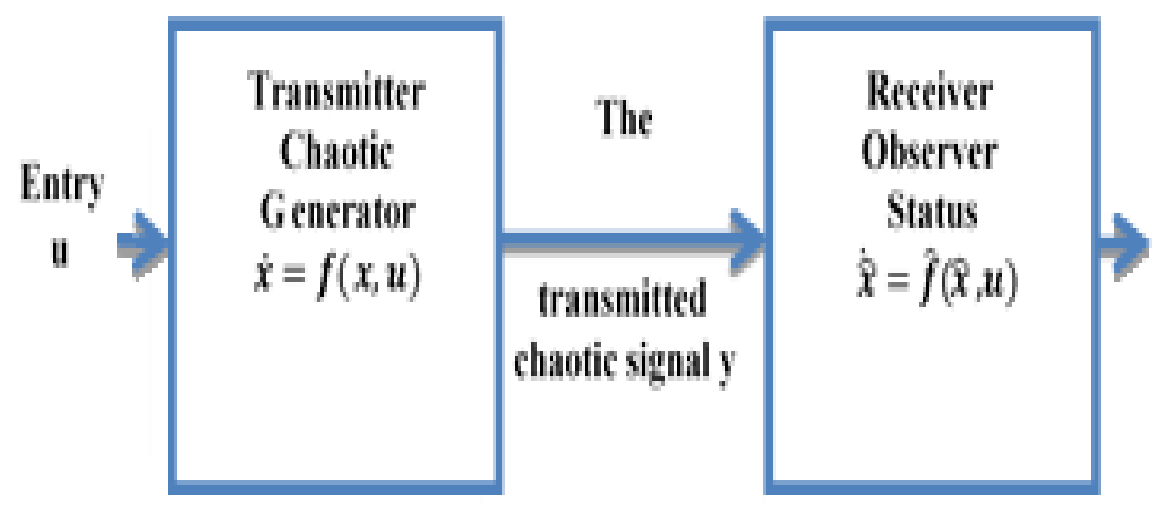

Figure 4. Principle of Master Slave Synchronization

In this section, we design a high observer design for the synchronization of two different 7D hyperchaotic systems. 
The 7D fourth-order hyperchaotic system is taken as the drive system, whose dynamics is given by:

$$
\begin{aligned}
& \dot{\mathrm{x}}_{1}=-\mathrm{a} \mathrm{x_{1 }}-20 \mathrm{x}_{5}-\mathrm{x}_{3} \mathrm{x}_{5} \mathrm{x}_{6} \mathrm{x}_{7} \\
& \dot{\mathrm{x}}_{2}=\mathrm{cx}_{2}-\mathrm{dx}_{6}+\mathrm{x}_{1} \mathrm{x}_{4} \mathrm{x}_{5} \mathrm{x}_{7} \\
& \dot{\mathrm{x}}_{3}=-\mathrm{ax}_{3}+\mathrm{ax}_{5}-\mathrm{g} \mathrm{x}_{1} \mathrm{x}_{2} \mathrm{x}_{4} \mathrm{x}_{6} \\
& \dot{\mathrm{x}}_{4}=-\mathrm{ax} \mathrm{x}_{4}+\mathrm{ex}_{1}+\mathrm{x}_{1} \mathrm{x}_{2} \mathrm{x}_{3} \mathrm{x}_{5} \\
& \dot{\mathrm{x}}_{5}=-\mathrm{ax}_{5}+\mathrm{ex}_{7}-\mathrm{x}_{1} \mathrm{x}_{2} \mathrm{x}_{3} \mathrm{x}_{4} \\
& \dot{\mathrm{x}}_{6}=-\mathrm{e} \mathrm{x}_{6}+\mathrm{ex}_{5}+\mathrm{x}_{2} \mathrm{x}_{3} \mathrm{x}_{4} \mathrm{x}_{5} \\
& \dot{\mathrm{x}}_{7}=-\mathrm{bx} \mathrm{x}_{7}+\mathrm{fx}_{2}-\mathrm{hx}_{1} \mathrm{x}_{4} \mathrm{x}_{5} \mathrm{x}_{6}
\end{aligned}
$$

The 7D six-order hyperchaotic system is also taken as the response system, whose dynamics is given by:

$$
\left\{\begin{array}{l}
\dot{\mathrm{z}}_{1}=15 \hat{\mathrm{z}}_{1}+20 \hat{\mathrm{z}}_{3}+0.785 \hat{\mathrm{z}}_{2} \hat{\mathrm{z}}_{3} \hat{\mathrm{z}}_{4} \hat{\mathrm{z}}_{5} \hat{\mathrm{z}}_{6} \hat{\mathrm{z}}_{7}-\theta \mathrm{d}_{\theta}^{-1} \mathrm{~S}_{1}^{-1} \mathrm{C}^{\prime}\left(\mathrm{C} \hat{\mathrm{z}}_{1}-\mathrm{x}_{1}\right) \\
\dot{\mathrm{z}}_{2}=-20 \hat{\mathrm{z}}_{1}-20 \hat{\mathrm{z}}_{2}-\hat{\mathrm{z}}_{1} \hat{\mathrm{z}}_{3} \hat{\mathrm{z}}_{4} \hat{\mathrm{z}}_{5} \hat{\mathrm{z}}_{6} \hat{\mathrm{z}}_{7}-\theta \mathrm{d}_{\theta}^{-1} \mathrm{~S}_{1}^{-1} \mathrm{C}^{\prime}\left(\mathrm{C} \hat{\mathrm{z}}_{2}-\mathrm{x}_{2}\right) \\
\dot{\hat{\mathrm{z}}}_{3}=20 \hat{\mathrm{z}}_{4}-15 \hat{\mathrm{z}}_{3}+20 \hat{\mathrm{z}}_{5}+\hat{\mathrm{z}}_{1} \hat{\mathrm{z}}_{2} \hat{\mathrm{z}}_{4} \hat{\mathrm{z}}_{5} \hat{\mathrm{z}}_{6} \hat{\mathrm{z}}_{7}-\theta \mathrm{d}_{\theta}^{-1} \mathrm{~S}_{1}^{-1} \mathrm{C}^{\prime}\left(\mathrm{C} \hat{\mathrm{z}}_{3}-\mathrm{x}_{3}\right) \\
\dot{\hat{\mathrm{z}}}_{4}=5 \hat{\mathrm{z}}_{4}-\hat{\mathrm{z}}_{1} \hat{\mathrm{z}}_{2} \hat{\mathrm{z}}_{3} \hat{\mathrm{z}}_{5} \hat{\mathrm{z}}_{6} \hat{\mathrm{z}}_{7}-\theta \mathrm{d}_{\theta}^{-1} \mathrm{~S}_{1}^{-1} \mathrm{C}^{\prime}\left(\mathrm{C} \hat{\mathrm{z}}_{4}-\mathrm{x}_{4}\right) \\
\dot{\hat{\mathrm{z}}}_{5}=-30 \hat{\mathrm{z}}_{5}+19 \hat{\mathrm{z}}_{3}+\hat{\mathrm{z}}_{1} \hat{\mathrm{z}}_{2} \hat{\mathrm{z}}_{3} \hat{\mathrm{z}}_{4} \hat{\mathrm{z}}_{6} \hat{\mathrm{z}}_{7}-\theta \mathrm{d}_{\theta}^{-1} \mathrm{~S}_{1}^{-1} \mathrm{C}^{\prime}\left(\mathrm{C} \hat{\mathrm{z}}_{5}-\mathrm{x}_{5}\right) \\
\dot{\hat{\mathrm{z}}}_{6}=10 \hat{\mathrm{z}}_{2}+3.9 \hat{\mathrm{z}}_{6}-\hat{\mathrm{z}}_{1} \hat{\mathrm{z}}_{2} \hat{\mathrm{z}}_{3} \hat{\mathrm{z}}_{4} \hat{\mathrm{z}}_{5} \hat{\mathrm{z}}_{7}-\theta \mathrm{d}_{\theta}^{-1} \mathrm{~S}_{1}^{-1} \mathrm{C}^{\prime}\left(\mathrm{C} \hat{\mathrm{z}}_{6}-\mathrm{x}_{6}\right) \\
\dot{\hat{\mathrm{z}}}_{7}=20 \hat{\mathrm{z}}_{3}-15 \hat{\mathrm{z}}_{7}+1.5 \hat{\mathrm{z}}_{1} \hat{\mathrm{z}}_{2} \hat{\mathrm{z}}_{3} \hat{\mathrm{z}}_{4} \hat{\mathrm{z}}_{5} \hat{\mathrm{z}}_{6}-\theta \mathrm{d}_{\theta}^{-1} \mathrm{~S}_{1}^{-1} \mathrm{C}^{\prime}\left(\mathrm{C} \hat{\mathrm{z}}_{7}-\mathrm{x}_{7}\right)
\end{array}\right.
$$

For the synchronization, the error e is defined as:

$$
\begin{gathered}
e_{1}=\hat{z}_{1}-x_{1}, e_{2}=\hat{z}_{2}-x_{2}, e_{3}=\hat{z}_{3}-x_{3}, e_{4}=\hat{z}_{4}-x_{4}, e_{5}=\hat{z}_{5}-x_{5}, e_{6}=\hat{z}_{6}- \\
x_{6}, e_{7}=\hat{z}_{7}-x_{7}
\end{gathered}
$$

\section{Results and Analysis}

In order to measure the performance of the proposed observer "response system", we employ the measure the impact of the error, suggested in Ogunnaike and Ray (1994), is the Integral Time-Weighted Squared Error (ITSE) dened in [15].

ITSE exhibits the advantage of heavy penalization of large errors at a long time; therefore, it is a good measure of resilience of the observer.

ITSE $=\int_{0}^{\infty} t \varepsilon^{2}$

With the observation error as $\varepsilon=x-\hat{x}$.

The parameter values as in the7D fourth-order hyperchaotic system case are assumed:

$a=15, b=5, c=0.5, d=25, e=10, f=4, g=0.1$ and $h=1.5$

For simulations, the initial conditions of the 7D fourth-order hyperchaotic system (8) are chosen as:

$$
x_{1}(0)=3, x_{2}(0)=1, x_{3}(0)=-1, x_{4}(0)=1, x_{5}(0)=2, x_{6}(0)=-2, x_{7}(0)=3
$$

Also, the initial conditions of the7D six-order hyperchaotic system (10) are chosen as:

$$
\hat{z}_{1}(0)=0, \hat{z}_{2}(0)=1, \hat{z}_{3}(0)=0, \hat{z}_{4}(0)=-2, \hat{z}_{5}(0)=1, \hat{z}_{6}(0)=0, \hat{z}_{7}(0)=4
$$


International Journal of Control and Automation

Vol. 10, No. 1 (2017)
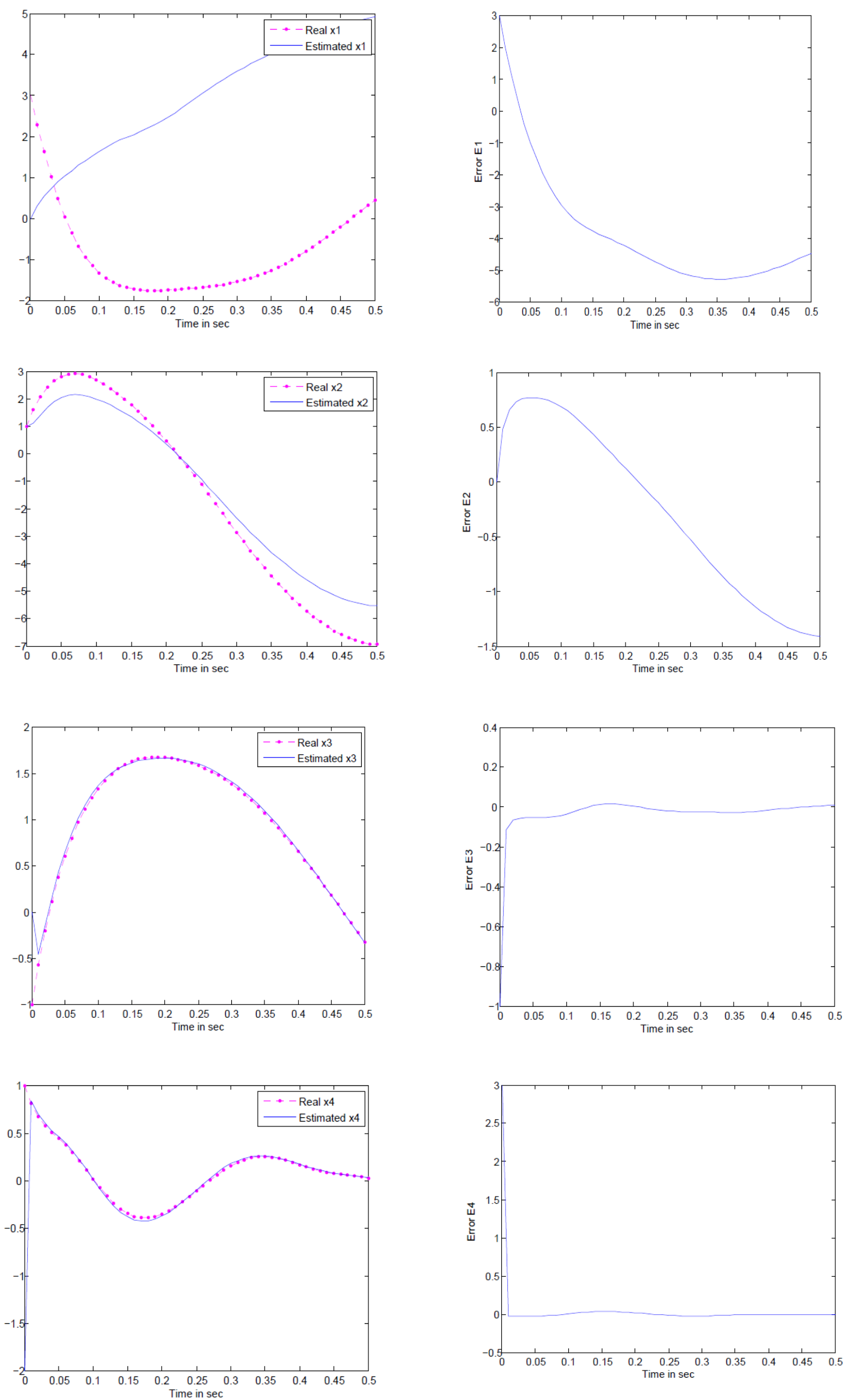

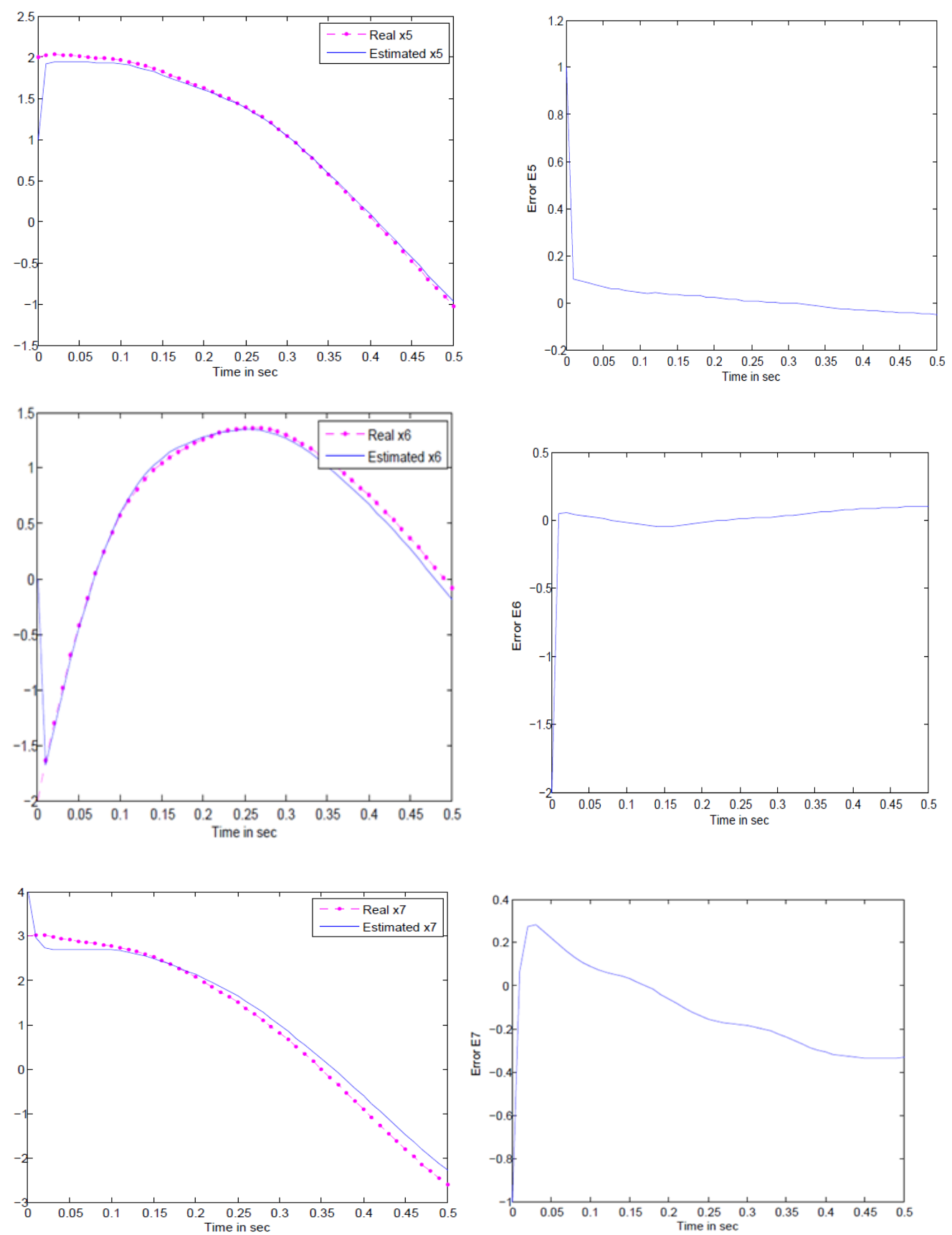

Figure 5. Response -Drive Synchronization Error of the 7D Fourth-Order and Six - Order Hyperchaotic System $(\theta=2)$

Figure 6. Synchronization Error $(\theta=2)$ 

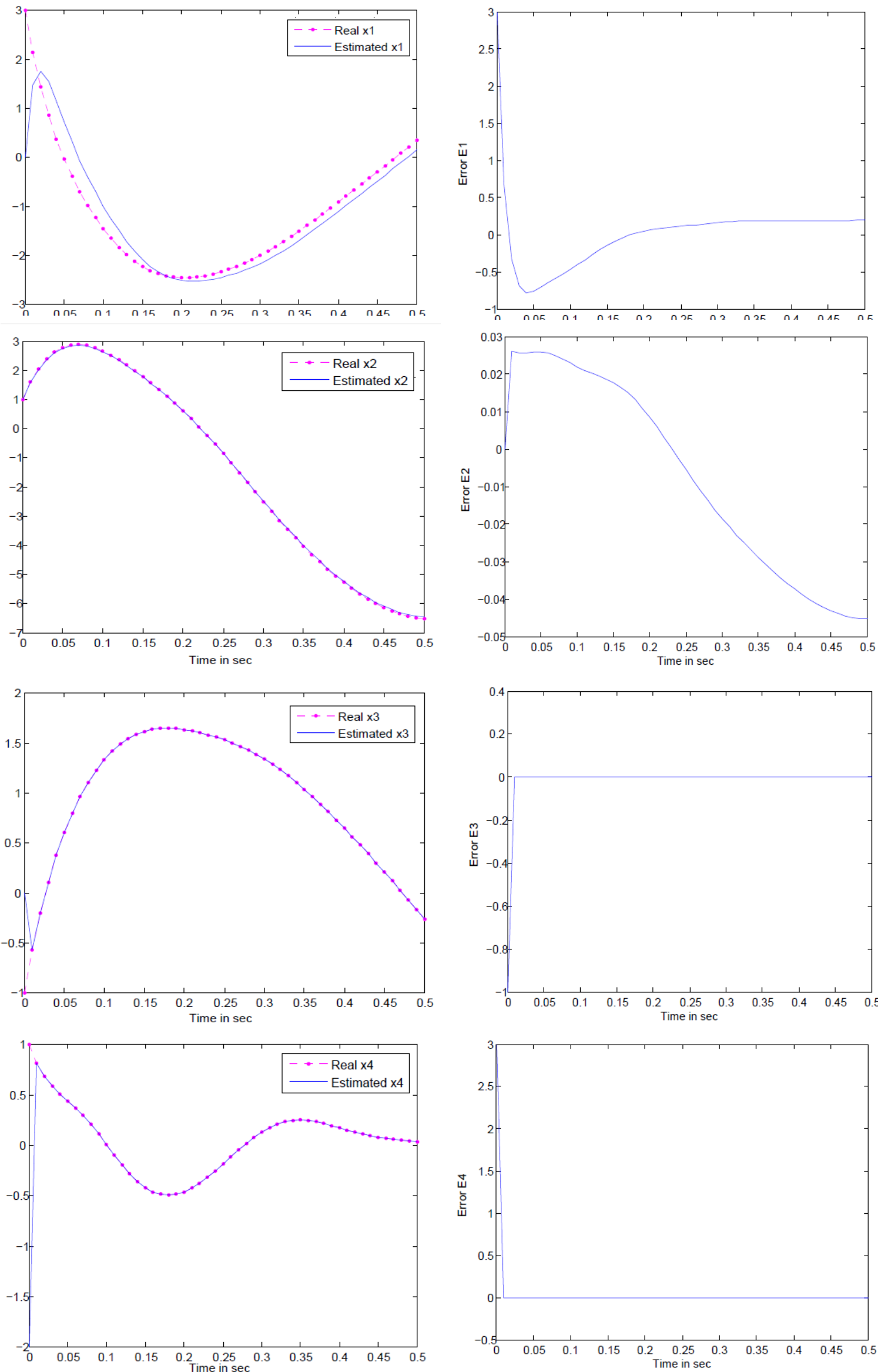

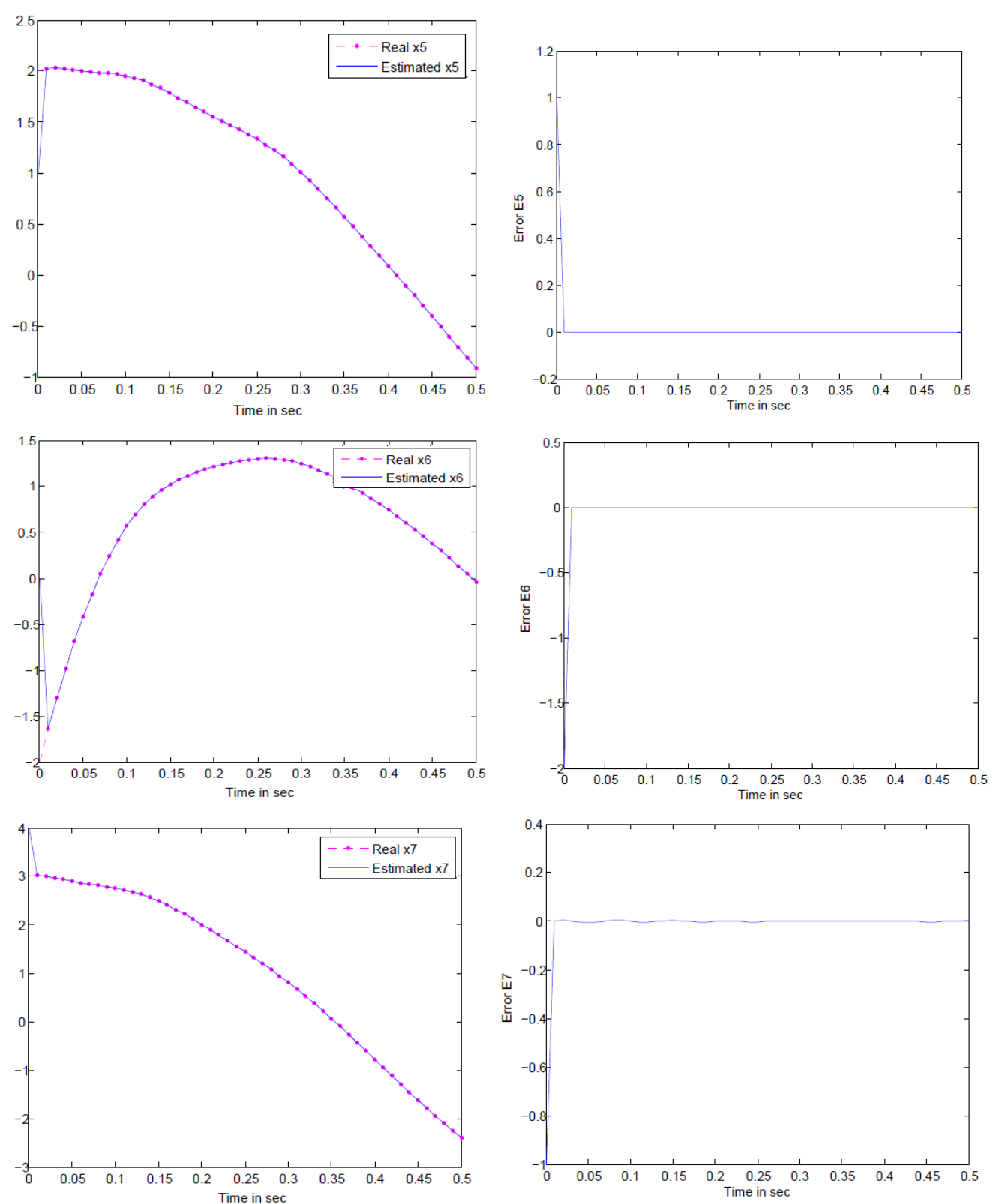

Figure 7. Response -Drive Synchronization Error of the 7D Fourth-Order and Six - Order Hyperchaotic System $(\theta=12)$

Figure 8. Synchronization Error

$$
(\theta=12)
$$

In Figures (6) and (8) is presented error for our drive-response hyperchaotic system. The seven values of guarantees asymptotic convergence to zero, especially for $\theta=12$.

From these figures, we can see that the synchronization error converge to zero and two different systems are indeed achieving chaos synchronization. 
Table 1. Corresponding Results of the ITSE Index For $\Theta=2$

\begin{tabular}{|c|c|c|c|c|c|c|c|c|c|c|c|}
\hline$t$ & $\mathbf{0}$ & 0.05 & 0.10 & 0.15 & 0.20 & 0.25 & 0.30 & 0.35 & 0.40 & 0.45 & 0.50 \\
\hline E1 & 3 & -1 & -2.80 & -3.7 & -4.2 & -4.6 & -5 & -5.2 & -5 & -4.80 & -4.5 \\
\hline E2 & 0 & 0.75 & 0.65 & 0.4 & 0.15 & $\begin{array}{c}- \\
0.15\end{array}$ & -0.5 & -0.8 & -1.1 & -1.3 & -1.4 \\
\hline E3 & $\overline{-}$ & $\begin{array}{c}- \\
0.05\end{array}$ & -0.02 & 0.01 & 0 & $\begin{array}{c}- \\
0.01\end{array}$ & -0.01 & $\begin{array}{c}- \\
0.01\end{array}$ & 0 & 0 & 0 \\
\hline E4 & 3 & 0 & 0.001 & 0.005 & 0.005 & 0 & 0 & 0 & 0 & 0 & 0 \\
\hline E5 & 1 & 0.05 & 0.025 & 0.02 & 0.001 & 0 & 0 & 0 & $\begin{array}{c}- \\
0.001\end{array}$ & $\begin{array}{c}- \\
0.0015\end{array}$ & $\begin{array}{c}- \\
0.025\end{array}$ \\
\hline E6 & $\overline{-}$ & 0.05 & 0 & -0.05 & 0 & 0.05 & 0.06 & 0.07 & 0.08 & 0.09 & 0.1 \\
\hline E7 & 1 & 0.25 & 0.1 & 0.05 & -0.05 & $\begin{array}{c}- \\
0.15\end{array}$ & $\begin{array}{c}- \\
0.175\end{array}$ & $\begin{array}{c}- \\
0.25\end{array}$ & -0.3 & -0.35 & -0.34 \\
\hline
\end{tabular}

Table 2. Corresponding Results of the ITSE Index For $\Theta=12$

\begin{tabular}{|c|c|c|c|c|c|c|c|c|c|c|c|}
\hline $\mathbf{E}$ & $\mathbf{0}$ & $\mathbf{0 . 0 5}$ & $\mathbf{0 . 1 0}$ & $\mathbf{0 . 1 5}$ & $\mathbf{0 . 2 0}$ & $\mathbf{0 . 2 5}$ & $\mathbf{0 . 3 0}$ & $\mathbf{0 . 3 5}$ & $\mathbf{0 . 4 0}$ & $\mathbf{0 . 4 5}$ & $\mathbf{0 . 5 0}$ \\
\hline $\mathbf{E 1}$ & 3 & -0.7 & -0.5 & -0.2 & 0 & 0.05 & 0.1 & 0.1 & 0.15 & 0.2 & 0.2 \\
\hline $\mathbf{E 2}$ & 0 & 0.025 & 0.022 & 0.018 & 0.01 & -0.005 & -0.02 & - & - & - & - \\
\hline E3 & -1 & 0 & 0 & 0 & 0 & 0 & 0 & 0 & 0 & 0 & 0 \\
\hline E4 & 3 & 0 & 0 & 0 & 0 & 0 & 0 & 0 & 0 & 0 & 0 \\
\hline E5 & 1 & 0 & 0 & 0 & 0 & 0 & 0 & 0 & 0 & 0 & 0 \\
\hline E6 & -2 & 0 & 0 & 0 & 0 & 0 & 0 & 0 & 0 & 0 & 0 \\
\hline E7 & 1 & 0 & 0 & 0 & 0 & 0 & 0 & 0 & 0 & 0 & 0 \\
\hline
\end{tabular}

The Table 1 and 2 show the estimated values of the corresponding performance index that decrease as $\theta$ increase.

For this, we have limited our simulation in $\theta=12$ because over this value the error was not a signified variation. 


\section{Conclusion}

Given that the mathematical model reflects the actual dynamics of our two new hyperchaotic systems, this research article shows the possibility of using a high gain observer to synchronize these systems.

In this paper, a seven-dimensional fourth-order hyperchaotic system and sevendimensional six-order hyperchaotic system were developped, and the following conclusions are obtained through the analysis of Lyapunov exponent and the system simulations:

The two seven-dimensional systems are hyperchaotic, they have at least two positive lyapunov exposent.

- The nonlinear high gain observer was derived the drive-response synchronization.

Numerical simulations are given to illustrate the effectiveness of the synchronization schemes derived in this paper of two 7D non-identical hyperchaotic systems.

The synchronization was approved in favor of the errors that tend to zero when the observer gain grows.

\section{References}

[1] T.L. Carroll and L.M.Pecora, "Synchronization in chaotic systems", Phys Rev Lett., vol. 64, (1990), pp. $821-4$.

[2] G. Chen and X. Dong, "From chaos to order. Singapore: World Scientific", (1998).

[3] V. Oppenheim, K. M. Cuomo and S. H. Strogatz, "Synchronization of Lorenz-based chaotic circuits with applications to communications", IEEE Trans. on CAS, part II, vol. 40, no. 10, (1993), pp. 626-633.

[4] H.K. Chen and C.I. Lee, "Anti-control of chaos in rigid body motion", Chaos, Solitons \& Fractals, vol. 21, (2004), pp. 957-65.

[5] S.N. Lagmiri, M. Amghar and N. Sbiti, "Synchronization between a new chaotic system and Rössler system by a high gain observer", IEEEXplore, (2014).

[6] Cafagna and G. Grassi, Int. J. Bifurcation and Chaos, vol. 13, (2003), pp. 2537.

[7] K. M. Cuomo, A. V. Oppenheim and S. H. Strogatz, IEEE Trans. Circuits Syst. vol. 40, no. 626, (1993).

[8] L. Kocarev, K. S. Halle, K. Eckert and L. O. Chua, Int. J. Bifurcation Chaos, vol. 2, no. 709, (1992).

[9] L. M. Pecora and T. L. Carroll, Phys. Rev. A, vol. 44, no. 2374, (1991).

[10] M.F Ahmad, M. Mamat, W.S MadaSanjaya and Z. Salleh, "Numerical simulation dynamical model of three-species food chain with lotka-volterra linear functional response", Journal of Sustainability Science and Management, vol. 6, no. 1, (2011), pp. 44-50.

[11] S.N.Lagmiri, H.El Mazoudi and N. Elalami, "Control of Lotka-Volterra three species system via a high gain observer design”, International Journal of Computer Applications (0975 8887), vol. 77, no. 15, (2013).

[12] V. Oppenheim, K. M. Cuomo and S. H. Strogatz, "Synchronization of Lorenz-based chaotic circuits with applications to communications", IEEE Trans. on CAS, part II, vol. 40, no. 10, (1993), pp. 626-633.

[13] R. Femat and G. Solis-Perales, "On the chaos synchronization phenomena", Phys. Lett. A, vol. 262, (1999).

[14] T. Carrol and L.M. Pecora, "Synchronization in chaotic systems", Phys. Rev. Lett., vol. 64, (1990).

[15] H.K. Chen and C.I. Lee, "Anti-control of chaos in rigid body motion", Chaos, Solitons\& Fractals vol. 21, (2004), pp. 957-65.

[16] V. Sundarapandian, "Adaptive control and synchronization of a generalized Lotka-Volterra system", International Journal of Bioinformatics \& Biosciences, vol. 1, no. 1, (2012), pp 1-12.

[17] T Carrol and L.M. Pecora, "Synchronization in chaotic systems", Phys. Rev. Lett., vol. 64, (1990).

[18] T. Carrol and L.M. Pecora, "Synchronization in chaotic systems", Phys. Rev. Lett., vol. 64, (1990).

[19] S.N.Lagmiri, H. ElMazoudi and N. Elalami, "High gain observer for Lotka Volterra system", Numerical Analysis Days and Optimization. Essaouira-Morocco, October 30 to November 1, (2013).

[20] S.N.Lagmiri, M.Amghar and N.Sbiti, "Synchronization between a new chaotic system and Rössler system by a high gain observer", 14th Mediterranean Microwave Symposium December 12-14, (2014), Marrakech, Morocco.

[21] S.N. Lagmiri, H.El Mazoudi and N. Elalami, "Synchronization of 4-d hyperchaotic qi system by high gain observer", International Conference on Structural Nonlinear Dynamics and Diagnosis (CSNDD'2014), May 19-21, Agadir, Morocco, (2014). 
International Journal of Control and Automation Vol. 10, No. 1 (2017) 